\title{
Abordagem supraclavicular da artéria subclávia direita para estabelecimento de circulação extracorpórea nas doenças da aorta
}

\author{
Hélio Antônio FABRI*, Cláudio Ribeiro da CUNHA*, Paulo César SANTOS*, \\ Daysi Mabel Pelegrinni CARIZZI* \\ *Da equipe de cirurgia cardiovascular do Hospital das Clínicas da Universidade Federal de Uberlândia.
}

RBCCV 44205-590

\section{Resumo}

Objetivo: Estabelecer a circulação extracorpórea através de canulação da artéria subclávia direita por meio de incisão supraclavicular.

Método: Foram estudados, no período de outubro de 2001 a março de 2002, quatro pacientes com diagnóstico de dissecção aguda de aorta tipo A de Stanford, sendo realizada em todos os casos canulação da artéria subclávia direita por via supraclavicular e realização de perfusão cerebral anterógrada durante o período de parada circulatória total.

Resultados: A artéria subclávia direita foi canulada diretamente e não houve nenhuma complicação neurovascular relacionada ao procedimento. A circulação extracorpórea foi mantida com fluxo adequado durante toda a operação. Houve 1 óbito hospitalar, não relacionado ao procedimento.

Discussão: Um dos passos mais importantes na melhora dos resultados operatórios é a perfusão sistêmica anterógrada, realizada através do sistema arterial e a subclávia para isso permite a perfusão da luz verdadeira. A abordagem supraclavicular nos proporciona um campo operatório adequado e facilidade para a realização da perfusão cerebral anterógrada que também é importante neste arsenal para uma redução da mortalidade operatória.

Conclusão: A incisão supraclavicular nos proporciona um acesso factível com boa exposição da artéria subclávia direita em uma região em que ela apresenta um bom calibre e com poucas estruturas adjacentes que poderiam ser lesadas. Além

\section{INTRODUÇÃO}

A dissecção aguda da aorta ascendente é uma emergência cirúrgica associada a significante morbidade e mortalidade, sendo a mais complexa doença do sistema cardiovascular, gerando graves conseqüências quando o disso, possibilita a canulação de forma direta e com boa posição da cânula arterial.

Descritores: Aorta, patologia. Aorta, cirurgia. Aneurisma dissecante, cirurgia. Circulação extracorpórea.

\section{Abstract}

Objetive: Establishment of cardiopulmonary bypass using the right subclavian artery with a supraclavicular approach.

Method: Between october 2001and march 2002, 4 patients were operated with the diagnosis of Stanford type A aortic dissection. The supraclavicular approach to the right subclavian artery was used for establishment of cardiopulmonary bypass in all of them.

Results: The right subclavian artery was canulated directly, without interposition of grafts. There was no neurovascular complication related to the procedure nor wound site complications. The hospital mortality was $25 \%$. The death was not procedure related.

Conclusion: The supraclavicular approach to the subclavian artery allows a good exposition of this artery in a region where it has enough size for direct canulation and establishment of cardiopulmonary bypass, with satisfactory canula position.

Descriptors: Aorta, pathology. Aorta, surgery. Aneurysm dissecting, surgery. Extracorporeal circulation.

tratamento não é realizado em tempo hábil ou este é feito de forma inadequada.

A partir do instante em que as doenças da aorta passaram a ser tratadas em um âmbito mais complexo e a aorta passou a ser encarada não simplesmente como um "tubo" intratorácico com a função de levar o sangue do coração para os

Trabalho realizado no Hospital das Clínicas da Universidade 
tecidos, a propedêutica e a terapêutica destas enfermidades passaram por grandes avanços, trazendo melhora substancial no resultado operatório e, conseqüentemente, na sobrevida dos pacientes.

Em 1955, DEBAKEY et al. ${ }^{(1)}$ relataram experiência inicial com o tratamento cirúrgico da dissecção da aorta. Estes autores descreveram a excisão da porção dilatada, união das camadas separadas e restauração da continuidade aórtica com anastomose término-terminal.

A primeira substituição da aorta ascendente com sucesso foi realizada por COOLEY \& DEBAKEY ${ }^{(2)}$, e tornouse o procedimento padrão para o tratamento das dissecções agudas da aorta ascendente.

A partir de então, o tratamento cirúrgico das afecções da aorta ascendente passou por vários avanços, sendo um deles o estabelecimento do local de canulação do sistema arterial, pois como há presença de dois lúmens é necessário que a perfusão seja adequada e realizada pela luz verdadeira da aorta para que os órgãos nobres sejam perfundidos de maneira satisfatória.

A artéria femoral tem sido o sítio preferido para a canulação do sistema arterial em operações naqueles pacientes com dissecções da aorta tipo A. Entretanto, a presença de doença arterial periférica, como aneurisma aortoilíaco, extensão distal da dissecção da aorta para as artérias femurais e doença aterosclerótica oclusiva grave do sistema aorto-ilíaco, pode contra-indicar esta opção, desta forma, deve-se procurar alternativas.

A canulação da aorta transapical através do ventrículo esquerdo é uma outra maneira de manutenção do fluxo arterial durante a circulação extracorpórea pela luz verdadeira com absoluta certeza, porém apresenta inconvenientes, como a necessidade de incisão na parede do ventrículo esquerdo, o que não é isento de complicações.

Recentemente, a abordagem da artéria axilar/subclávia tem sido realizada com sucesso para o estabelecimento da circulação extracorpórea nos casos de dissecção aguda de aorta e em alguns casos de aneurisma verdadeiro de aorta ascendente com envolvimento do arco e porção inicial da aorta descendente. As artérias subclávias, tanto direita quanto esquerda, podem ser usadas para essa finalidade, e a via de acesso é realizada normalmente por via infraclavicular. No nosso estudo realizamos a abordagem da artéria subclávia direita por via supraclavicular.

\section{MÉTODO}

No período compreendido entre novembro de 2001 e março de 2002, no Serviço de Cirurgia Cardíaca do Hospital de Clínicas da FAEPU da Universidade Federal de Uberlândia foram operados quatro pacientes para correção de dissecção de aorta, sendo dois com dissecção aguda e dois com dissecção crônica. Em todos, a artéria subclávia foi utilizada, de forma eletiva, como sítio para canulação arterial para estabelecimento da circulação extracorpórea.
Três pacientes eram do sexo masculino e 1 do feminino. A idade média era de 42,75 anos. Apenas 1 paciente apresentava o estigma da síndrome de Marfan. Os antecedentes pessoais patológicos são apresentados na Tabela 1 .

Tabela 1. Antecedentes pessoais

\begin{tabular}{lc}
\hline Antecedentes & $\%$ \\
\hline Sexo feminino & 25 \\
Hipertensão arterial sistêmica & 75 \\
Tabagismo & 50 \\
Diabetes mellitos & $-\overline{25}$ \\
Síndrome de Marfan & 25 \\
Claudicação intermitente & 25 \\
Aneurisma de aorta & - \\
Angina & - \\
Insuficiência renal & - \\
DPOC &
\end{tabular}

Quanto à forma de apresentação, o sintoma mais comum foi a dor torácica, presente em $75 \%$ dos casos. Nenhum dos pacientes apresentava sinais de isquemia cerebral, miocárdica ou de vísceras abdominais. As condições clínicas dos pacientes são apresentadas na Tabela 2.

Tabela 2. Condição clínica

\begin{tabular}{lc}
\hline Sintomatologia & $\%$ \\
\hline Dor torácica & 75 \\
Síncope & - \\
Hipotensão & $\overline{75}$ \\
Insuficiência aórtica & 50 \\
Derrame pericárdico & - \\
Tamponamento cardíaco & - \\
Ressuscitação cardiopulmonar & - \\
Isquemia miocárdica & - \\
Isquemia cerebral & - \\
Isquemia medular & - \\
Isquemia de vísceras abdominais & - \\
Isquemia de membros inferiores &
\end{tabular}

Todos os pacientes foram submetidos a radiografia de tórax e ecocardiograma transtorácico. A tomografia de tórax com emprego de contraste endovenoso e a angiografia foram empregadas em $75 \%$ dos pacientes. A propedêutica empregada na avaliação da dissecção da aorta está sumarizada na Tabela 3.

Tabela 3. Avaliação diagnóstica dos pacientes com dissecção de aorta

\begin{tabular}{lr}
\hline Exame & $\%$ \\
\hline Radiografia de tórax & 100 \\
Ecocardiograma transtorácico & 100 \\
Ecocardiograma transesofágico & 50 \\
Tomografia computadorizada & 75 \\
Ressonância magnética & $\overline{75}$ \\
Angiografia & \\
\hline
\end{tabular}

A técnica cirúrgica empregada foi particularizada em cada caso, de acordo com as características da dissecção encontradas no intra-operatório e a presença de acometimento valvar ou coronariano associado. 
Em um paciente com diagnóstico de dissecção aguda de aorta e insuficiência valvar aórtica discreta a moderada, a aorta ascendente foi substituída por um enxerto de dacron. A anastomose distal do enxerto na aorta foi feita sob parada circulatória total em hipotermia profunda, e a anastomose proximal foi feita em posição supracoronariana, em circulação extracorpórea sob hipotermia moderada. $\mathrm{O}$ tratamento da insuficiência aórtica consistiu apenas na ressuspensão das comissuras da valva aórtica.

Em um segundo paciente com diagnóstico de dissecção aguda de aorta e insuficiência coronária crônica (lesão obstrutiva de $90 \%$ de primeiro ramo marginal esquerdo) foi feita a substituição da aorta ascendente por um enxerto de dacron por técnica semelhante à descrita no caso anterior, associada à revascularização do miocárdio. Terminada a substituição da aorta ascendente, ainda em circulação extracorpórea e hipotermia moderada, procedeu-se à confecção da ponte com enxerto de veia safena, sendo a porção distal da veia anastomosada ao primeiro ramo marginal esquerdo e a proximal ao enxerto de dacron que substituiu a aorta ascendente.

Um terceiro paciente com diagnóstico de síndrome de Marfan, ectasia anuloaórtica associada à insuficiência valvar aórtica importante e dissecção crônica de aorta, foi submetido à substituição da aorta ascendente e raiz da aorta por enxerto composto (tubo de dacron com prótese mecânica de duplo folheto). A anastomose da porção distal do tubo na aorta foi feita sob parada circulatória total e hipotermia profunda, e a anastomose proximal e o reimplante dos óstios coronarianos foram feitos sob hipotermia moderada em circulação extracorpórea.

O quarto paciente havia sido submetido a correção de dissecção aguda de aorta com substituição da aorta ascendente por enxerto de dacron e posicionamento de uma prótese endovascular auto-expansível na aorta descendente, imediatamente após a origem da artéria subclávia esquerda. Este paciente tinha o diagnóstico de insuficiência aórtica severa pós-correção de dissecção da aorta. Foi submetido à canulação eletiva da artéria subclávia devido à persistência da dissecção da aorta na porção descendente. A proposta cirúrgica era de se fazer a substituição da valva aórtica por prótese mecânica, com pinçamento da aorta ascendente em circulação extracorpórea, sob hipotermia moderada. Após a aortotomia foi observada dissecção da aorta ascendente, imediatamente abaixo da sutura da aorta ascendente ao tubo de dacron, estendendo-se até o plano valvar, provocando o desabamento da valva aórtica. Foi feita a substituição da valva aórtica por prótese mecânica de duplo folheto e fechamento do orifício de entrada da dissecção com retalho de pericárdio bovino.

O tempo médio de parada circulatória total foi de 31,6 min (20 a 35 min, o tempo médio de circulação extracorpórea foi de $191 \mathrm{~min}$ (160 a $240 \mathrm{~min}$ ) e o tempo de médio de anóxia miocárdica foi de $152 \mathrm{~min}$ (140 a $179 \mathrm{~min}$ ).

\section{Técnica de abordagem da artéria subclávia}

A abordagem da artéria subclávia realiza-se através da via supraclavicular (Figura 1).

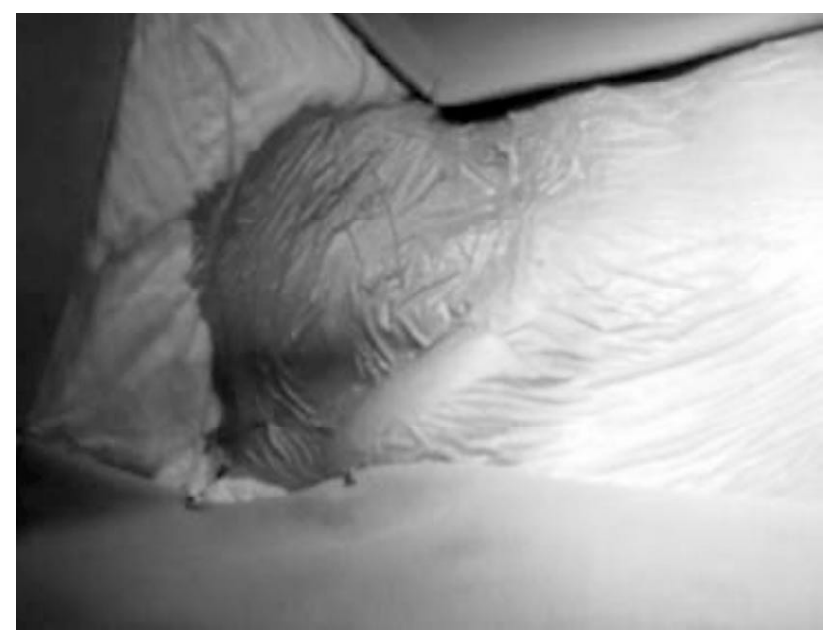

Fig. 1 - Campo operatório preparado para dissecção da artéria subclávia

Faz-se uma incisão de cerca de $6 \mathrm{~cm}$, na base do trígono posterior do pescoço, isto é, na fossa supraclavicular direita, iniciando-se na borda posterior do músculo esternocleidomastóideo com extensão em direção à inserção do músculo trapézio, paralela à clavícula, cerca de 1,5 a 2 cm acima desta. A incisão atravessa a pele, a fáscia cervical superficial, o platisma e a fáscia cervical profunda. A veia jugular externa cruza a parte medial da artéria subclávia, podendo ser necessária a sua ligadura ou de suas tributárias (veia cervical transversa, veia jugular anterior e veia escapular transversa) para facilitar a exposição da artéria. A terceira porção da artéria subclávia é facilmente encontrada no tecido celular frouxo logo abaixo da fáscia cervical profunda, em relação com a face posterior da borda lateral do músculo escaleno anterior, medialmente e com o plexo braquial, lateralmente. Neste ponto ela é isolada, e para melhor exposição, procede-se à secção do músculo escaleno anterior, próximo a sua inserção na primeira costela, com o cuidado de identificar e preservar o nervo frênico que está relacionado à borda medial deste músculo. Deste modo, a exposição de uma extensão adequada da artéria subclávia (cerca de 3 a $4 \mathrm{~cm}$ ) é possível.

A artéria é, em seguida, enlaçada com duas fitas cardíacas. A primeira é colocada na porção mais distal da artéria junto à clavícula, na porção da artéria subclávia que se localiza sobre a primeira costela. A segunda é colocada no segmento proximal, imediatamente distal à origem dos ramos da artéria subclávia: vertebral, torácica interna, tronco tireocervical e costocervical.

Após o preparo da artéria subclávia descrito acima, procedese à toracotomia mediana com esternotomia (Figura 2) e preparo para canulação venosa (veias cavas superior e inferior). 


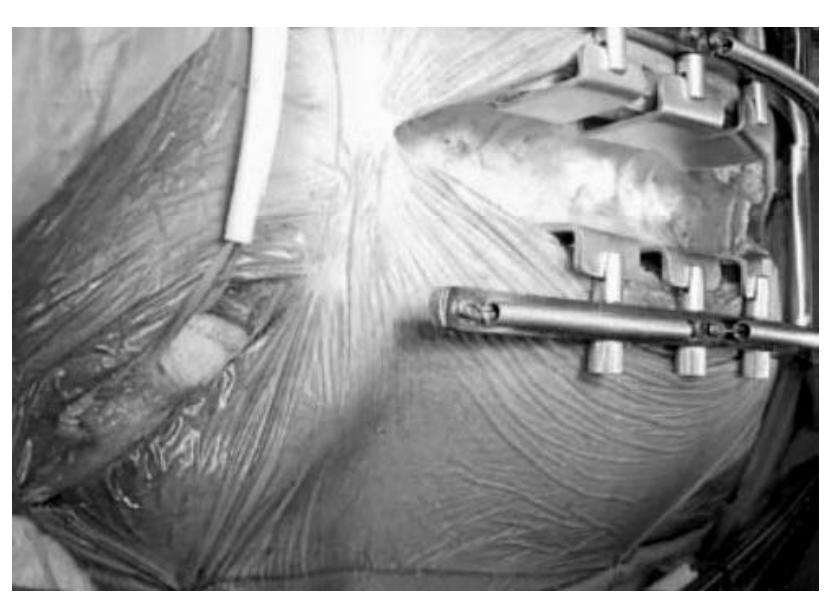

Fig. 2 - Visão conjunta da cervicotomia supraclaviculare da toracotomia

Concomitantemente, procede-se à dissecção e ao reparo do tronco braquiocefálico. Terminado este preparo, partese para a canulação arterial e, em seguida, venosa.

Após heparinização sistêmica ( $4 \mathrm{mg} / \mathrm{kg}$ de peso de heparina não fracionada por via endovenosa), a artéria subclávia previamente exposta e reparada é pinçada proximal e, em seguida, distalmente no local onde foram colocadas as fitas. Faz-se uma arteriotomia transversa e introduz-se uma cânula arterial reta (originalmente produzida para canulação da artéria femoral) 18 ou 20 Fr e aperta-se a fita que enlaça a porção proximal da subclávia com um torniquete, impedindo o refluxo de sangue entre a artéria e a cânula (Figura 3).

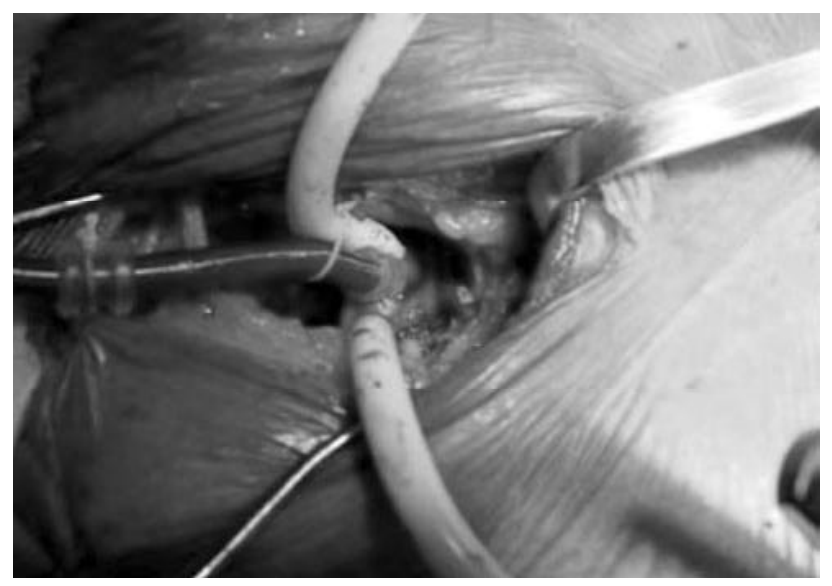

Fig. 3 - Artéria subclávia canulada

Fixa-se a cânula adequadamente ao campo cirúrgico (à pele), testa-se o refluxo de sangue e a resistência ao fluxo e conecta-se a cânula arterial ao sistema de circulação extracorpórea. A seguir, canulam-se as veias cavas. Durante a circulação extracorpórea, a região da dissecção da artéria subclávia é coberta com compressa embebida em soro fisiológico aquecido. A circulação extracorpórea é estabelecida com o uso de um oxigenador de membrana, e mantida com um fluxo de 2,2 1/ $/ \mathrm{min} / \mathrm{m}^{2}$ de superfície corpórea.
A adição de sangue ao volume utilizado para o preenchimento do circuito da circulação extracorpórea foi feita de acordo com cálculos considerando-se o hematócrito do paciente e a diluição prevista.

A hipotermia profunda é obtida através do uso de um permutador de calor incorporado ao sistema de circulação extracorpórea, e o paciente é resfriado até que a temperatura nasofaríngea do paciente atinja $18^{\circ} \mathrm{C}$. Imediatamente antes da parada circulatória total, são administrados dexametasona na dose de $10 \mathrm{mg}$ e tiopental na dose de $1 \mathrm{~g}$.

Imediatamente após o início da parada circulatória total, a origem do tronco braquiocefálico é ocluída e inicia-se a infusão de sangue oxigenado hipotérmico $\left(15 \mathrm{a} 18^{\circ} \mathrm{C}\right)$ pela cânula posicionada na artéria subclávia, com um fluxo de 300 a $500 \mathrm{ml}$ por minuto, mantendo-se livre a drenagem da linha venosa conectada à veia cava superior. Deste modo, durante todo o período de parada circulatória total, o território irrigado pelos ramos das artérias subclávia e carótida comum direita continuará perfundido.

Quando do restabelecimento da circulação extracorpórea, abre-se o cadarço oclusor da origem do tronco braquiocefálico e, gradativamente, aumenta-se o fluxo pela linha arterial até o restabelecimento do fluxo total da circulação extracorpórea.

Terminada a circulação extracorpórea, antes da reversão da anticoagulação com a protamina, retira-se a cânula e procede-se à arteriorrafia subclávia com sutura contínua com fio monofilamentar de polipropileno 6-0. Após a revisão da hemostasia, aproxima-se o platisma com sutura contínua absorvível (poligalactina 3-0) e sutura-se a pele com sutura contínua absorvível (poligalactina 4-0). Não há necessidade de drenagem.

\section{RESULTADO}

\section{Aplicabilidade da técnica}

A canulação da artéria subclávia foi possível em todos os casos. Em todos os pacientes a dissecção foi feita sem maiores dificuldades, sendo possível a exposição de um segmento adequado da artéria para canulação. A artéria subclávia estava livre de processo aterosclerótico ou extensão da dissecção em todos os casos.

\section{Morbidade}

Todos os pacientes sobreviveram à operação. Nenhum deles apresentou trombose ou dissecção da artéria subclávia, lesão do plexo braquial, do nervo frênico ou isquemia do membro superior direito. A cicatrização da ferida resultante da abordagem da artéria subclávia via supraclavicular transcorreu sem problemas em todos os pacientes, não sendo verificada infecção ou deiscência.

Nenhum paciente foi reoperado por sangramento ou derrame pericárdico.

Apenas um paciente apresentou necessidade de suporte inotrópico prolongado com quadro de baixo débito cardíaco. 
Este paciente evoluiu com um quadro de septicemia, de provável origem pulmonar e, posteriormente, insuficiência de múltiplos órgãos, culminando com o óbito no décimo primeiro dia de pós-operatório.

Um paciente apresentou quadro de acidente vascular cerebral isquêmico no pós-operatório imediato, apresentando quadro de hemiparesia direita e diminuição do nível de consciência. Evoluiu com dificuldade de desmame de ventilação mecânica, sendo necessária traqueostomia no décimo quinto dia de pós-operatório. No décimo sétimo dia de pós-operatório apresentou quadro de instabilidade hemodinâmica associado a anemia aguda e foi diagnosticado hematoma de retroperitôneo. Foram feitos reposição volêmica e acompanhamento clínico com melhora progressiva. O paciente teve alta no vigésimo oitavo dia de pós-operatório em boas condições clínicas, com discreta paresia de membro superior direito.

\section{Mortalidade}

Um (25\%) óbito ocorreu no décimo primeiro dia de pósoperatório, como resultado de falência de múltiplos órgãos secundária à septicemia com provável foco infeccioso pulmonar.

\section{COMENTÁRIOS}

As afecções da aorta, primordialmente as dissecções, são doenças que têm como ponto de partida o acometimento da aorta e seus ramos, porém na grande maioria das vezes estende-se para vários órgãos, como rins, cérebro e pulmões e, além disso, a cirurgia corretiva afeta estes mesmos órgãos. Portanto, ao se corrigir uma doença desta magnitude, devese levar em consideração as conseqüências das técnicas empregadas, sendo importante o manuseio correto da falsaluz no intra-operatório e a manutenção de uma perfusão adequada pela luz verdadeira para os órgãos nobres, além de uma satisfatória proteção cerebral, que só poderão ser conseguidos através de um fluxo arterial sistêmico adequado.

A utilização da artéria femoral tem sido proposta como uma opção de canulação do sistema arterial, principalmente nos casos de dissecção de aorta ${ }^{(3)}$. Entretanto, a perfusão sistêmica é realizada por via retrógrada e isso pode acarretar alguns inconvenientes, como embolia cerebral retrógrada. Além disso, nos casos com doença aorto-ilíaca aterosclerótica grave e doença aterosclerótica periférica severa torna-se proibitiva a canulação da artéria femural. Há ainda complicações, não muito raras, inerentes ao procedimento, como isquemia de membros inferiores e necessidade de realização de arterioplastias para correção do sítio de inserção da cânula arterial, linfocele e infecção local, principalmente em obesos ${ }^{(4)}$.

O fator mais importante quando se realiza a perfusão pela artéria femural é a adequação do fluxo para a luz verdadeira, já que em $42 \%$ dos casos o fluxo é realizado através da luz falsa, e quando isso ocorre, os órgãos nobres ficam mal perfundidos, acarretando uma maior morbidade, e há risco maior de sangramento da linha de sutura distal e maior probabilidade de rotura da aorta distal.

Para minimizar esta intercorrência é de fundamental importância que o fluxo da circulação extracorpórea seja mantido para a luz verdadeira, e para isso têm-se buscado outros sítios para a realização deste procedimento.

Naqueles casos com grande extensão do acometimento da dissecção em todo o segmento da aorta e seus ramos, pode-se usar a canulação da aorta ascendente por via transventricular, pelo ápice do ventrículo esquerdo ${ }^{(5)}$. Porém existem alguns inconvenientes, tais como a realização de mais uma incisão numa musculatura friável e risco de lesão nas válvulas da valva aórtica.

O uso da artéria axilar ou subclávia tem sido estudado por vários autores e com bons resultados, entre eles, NERI et al. ${ }^{(6)}$ e BARIBEAU et al. ${ }^{(7)}$. Estes últimos estudaram 29 pacientes num período de 3 anos com bons resultados. KATOH et al. ${ }^{\left({ }^{()}\right.}$usaram a artéria axilar direita para canulação do sistema arterial e WHITLARK et al. ${ }^{(9)}$ descreveram dados de 13 pacientes sem complicações referentes ao procedimento.

Em nosso estudo, analisamos quatro pacientes operados com o diagnóstico de dissecção aguda de aorta, nos quais realizou-se a canulação da artéria subclávia direita, através de incisão supraclavicular sem a interposição de enxerto. Outros autores como NERI et al. ${ }^{(6)}$, WHITLARK et al. ${ }^{(9)}$, BARIBEAU et al. ${ }^{(10)}$ e SABIK et al. ${ }^{(11)}$ propuseram inicialmente a canulação da artéria subclávia por via infraclavicular e encontraram alguns problemas, como nos pacientes obesos em que o uso deste local era praticamente impossível, e a exposição da artéria era um pouco limitada pelos ramos do plexo braquial e pela veia axilar que encontrase anteriormente à artéria, além dela possuir um calibre menor nesta porção. A profundidade do campo operatório dificulta de certa forma a colocação da cânula, prejudicando o bom funcionamento da perfusão sistêmica anterógrada. Na abordagem supraclavicular, a exposição da artéria subclávia torna-se ainda mais fácil após a realização da esternotomia e colocação do afastador do esterno, pois a sua abertura desvia a clavícula inferiormente, melhorando a exposição da artéria, o que não acontece na abordagem infraclavicular.

A canulação direta da artéria subclávia ou axilar tem sido proposta por alguns autores como SABIK et al. ${ }^{(11)}$ e LEYH et al. ${ }^{(12)}$. Este últimos, observaram $5,7 \%$ de incidência de injúria neurovascular em 35 pacientes na Cleveland Clinic, incluindo um caso de trombose de artéria axilar. Outros autores, com o objetivo de evitar o contato direto da cânula arterial rígida com o tecido frágil da artéria subclávia, usaram uma interposição de enxerto de dacron, com o objetivo de evitar tais complicações ${ }^{(10,13)}$.

Em nosso estudo, realizamos a canulação direta da artéria subclávia, pois esta exposição permite encontrarmos um vaso mais calibroso a este nível e a maneira pela qual é exposta a artéria, nos proporciona uma forma confortável 
de procedermos à canulação sem danos a sua parede, pois é possível realizar uma arteriotomia proporcional ao calibre da cânula, com probabilidade mínima de lesão da parede do vaso. Não se observou nenhuma complicação vascular do membro superior direito, fato comprovado pela realização de ecodoppler em todos os pacientes. Em todos os casos a arteriotomia foi realizada de forma direta, sem a necessidade de colocação de enxerto.

$\mathrm{Na}$ abordagem infraclavicular alguns reparos anatômicos são importantes e devem ser salientados, como por exemplo, a veia axilar direita cruza anteriormente a artéria a este nível e deve ser reparada e rechaçada para uma adequada abordagem da artéria e além disso, os fascículos do plexo braquial devem ser deslocados para uma perfeita visibilização do vaso, o que pode acarretar danos neurovasculares transitórios ou até permanentes.

$\mathrm{Na}$ incisão supraclavicular é necessária somente a liberação do tecido celular frouxo que envolve o vaso, não havendo necessidade de manipulação do plexo braquial e nem da veia subclávia, minimizando o risco de lesões neurovasculares. Algumas vezes é necessária a secção completa do músculo escaleno anterior, porém sem conseqüências funcionais significativas.

A perfusão realizada através da artéria subclávia direita proporciona um fluxo adequado para os vasos da base, como demonstrado pelos estudos de GERDES et al. ${ }^{(14)}$, que elaboraram um modelo experimental e realizaram teste "in vitro", com a simulação de vários diâmetros da artéria subclávia direita.

Neste experimento, media-se o fluxo e pressão de perfusão, concluindo que a perfusão sistêmica anterógrada realizada neste sítio proporciona um fluxo satisfatório. Isto nos mostra que, a perfusão sistêmica anterógrada pode ser realizada de maneira segura e ainda permite a realização da perfusão cerebral anterógrada, diminuindo o risco da embolia cerebral retrógrada, quando se realiza perfusão sistêmica retrógrada, pois esta última pode ocasionar uma oclusão dos vasos da base pelo "flap" intimal e ainda um risco de $42 \%$ de perfusão pela falsa luz.

O fato da canulação da artéria subclávia direita nos proporcionar um fluxo anterógrado satisfatório permite inferir que pode-se realizar perfusão cerebral anterógrada, nas operações sobre o arco aórtico e aorta descendente proximal. A proteção cerebral é um passo importante nas operações sobre o arco aórtico, e em particular nos casos de dissecção aguda da aorta cuja aorta ascendente é estudada em sua totalidade inclusive arco aórtico à procura da lesão intimal para que possa ser corrigida. Durante o período de parada circulatória total, a proteção cerebral faz-se fundamentalmente pela hipotermia profunda.

No entanto, a proteção conferida pela hipotermia sem perfusão cerebral é limitada pelo tempo. Assim, justifica-se a utilização da perfusão cerebral retrógrada através da veia cava superior ${ }^{(15)}$ ou anterógrada através da canulação seletiva dos vasos da base ${ }^{(16)}$ ou através da artéria subclávia já canulada.

Ao proceder-se a parada circulatória total e hipotermia profunda, a perfusão cerebral foi mantida através da subclávia direita com garroteamento do tronco braquicefálico. Não se usou pinçamento pelo risco de dissecção do tronco no local do pinçamento ${ }^{(17)}$. Existem outras formas de realização da perfusão cerebral anterógrada, como por exemplo, a interposição de enxerto sintético em um dos vasos da base, porém isto dispensa mais uma sutura e mais uma abordagem diferente, sendo que a canulação da artéria subclávia direita serve com o propósito de perfusão sistêmica e cerebral anterógrada.

Em decorrência dos avanços do manuseio anestésico e técnicas operatórias, o tratamento da dissecção aguda da aorta nas últimas décadas tem sofrido um impacto significativo na redução da mortalidade, conseqüência principalmente do enfoque da tática cirúrgica na correção total da doença e na perfusão adequada dos órgãos no intra-operatório através de um fluxo adequado para a luz verdadeira, e neste caso a canulação da artéria subclávia merece um destaque especial na medida em que assegura um fluxo sistêmico adequado.

Portanto, a canulação da artéria subclávia direita por via supraclavicular parece ser uma abordagem segura e reprodutível do aspecto técnico com baixo índice de complicações e vantagens em relação à abordagem convencional, infraclavicular.

\section{CONCLUSÕES}

A incisão supraclavicular parece proporcionar um acesso factível com boa exposição da artéria subclávia direita em uma região em que ela apresenta um bom calibre e com poucas estruturas adjacentes que poderiam ser lesadas e, além disso, possibilita a canulação de forma direta e com boa posição da cânula arterial.

\section{REFERÊNCIAS BIBLIOGRÁFICAS}

1. DeBakey ME, Cooley DA, Creech O - Surgical considerations of dissecting aneurysm of the aorta. Ann Surg 1955; 142:586-90.

2. Cooley DA \& DeBakey ME - Ressection of the entire ascending aorta in fusiform aneurysm using temporary cardiac bypass. JAMA 1956; 162:1158-62.

3. Borst HG, Schaudig A, Rudolph W - Arteriovenous fistula of the aortic arch: repair during deep hypothermia and circulatory arrest. J Thorac Cardiovasc Surg 1964; 48:443-8.

4. Gates JD, Bichell DP, Rizzo RJ, Couper GS, Donaldson MC Thigh ischemia complicating femoral vessel cannulation for cardiopulmonary bypass. Ann Thorac Surg 1996; 61:730-3.

5. Flege Jr. JB \& Aberg T - Transventricular aortic cannulation for repair of aortic dissection. Ann Thorac Surg 2001; 72:955-6. 
6. Neri E, Masseti M, Capannini G et al. - Axillary artery cannulation in type A aortic dissection operations. J Thorac Cardiovasc Surg 1999; 118:324-9.

7. Baribeau YR, Westbrook BM, Charlesworth DC, Maloney CT - Arterial inflow via an axillary artery graft for the severely atheromatous aorta. Ann Thorac Surg 1998; 66:33-7.

8. Katoh T, Gohra H, Hamano K, Takenaka H, Zempo N, Esato K - Right axillary cannulation in the left thoracotomy for thoracic aortic aneurysm. Ann Thorac Surg 2000;70:311-3.

9. Whitlark JD, Goldman SM, Sutter FP - Axillary artery cannulation in acute ascending aortic dissections. Ann Thorac Surg 2000; 69:1127-9.

10. Baribeau YR, Westbrook BM, Charlesworth DC - Axillary cannulation: first choice for extra-aortic cannulation and brain protection. J Thorac Cardiovasc Surg 1999; 118:1153-4.

11. Sabik JF, Lytle BW, McCarthy PM, Cosgrove DM - Axillary artery: an alternative site of arterial cannulation for patients with extensive aortic and peripheral vascular disease. J Thorac Cardiovasc Surg 1995; 109: 885-91.
12. Leyh RG, Bartels C, Nötzold A, Sievers HH - Management of porcelain aorta during coronary artery bypass grafting. Ann Thorac Surg 1999; 67:986-8.

13. Bichell DP, Balaguer JM, Aranki SF et al. - Axilloaxillary cardiopulmonary bypass: a practical alternative to femorofemoral bypass. Ann Thorac Surg 1997; 64:702-5.

14. Gerdes A, Jouber-Hübner E, Esders K, Sievers HH Hydrodynamics of aortic arch vessels during perfusion through the right subclavian artery. Ann Thorac Surg 2000; 69:1425-30.

15. Ueda Y, Miki S, Kusuhara K, Okita Y, Tahata T, Yamanaka K Deep hypothermic systemic circulatory arrest and continuous retrograde cerebral perfusion for surgery of aortic arch aneurysm. Eur J Cardiothorac Surg 1992; 6:36-41.

16. Bachet J, Guilmet D, Goudot B et al. - Antegrade cerebral perfusion with cold blood: a 13-year experience. Ann Thorac Surg 1999; 67:1874-94.

17. Imanaka K, Kyo S, Tanabe H, Ohuchi H, Asano H, Yokote Y - Fatal intraoperative dissection of the innominate artery due to perfusion through the right axillary artery. $\mathrm{J}$ Thorac Cardiovasc Surg 2000; 120:405-6. 